\title{
Comparison between Flat Spiral and Serpentine Differential Delay Lines on TDR and TDT
}

\author{
Wei-De Guo, Guang-Hwa Shiue, and Ruey-Beei Wu. \\ Department of Electrical Engineering and Graduate Institute of Communication Engineering, \\ National Taiwan University, Taipei, Taiwan 10617, R.O.C. \\ Tel: $+886-2-23635251 \times 340 \quad$ Fax: $+886-2-23638247$ \\ E-mail: rbwu@ew.ee.ntu.edu.tw
}

\begin{abstract}
In contrast to the commonly employed single delay lines, differential delay lines may alleviate the occurrence of crosstalk and improve the signal integrity. This paper investigates the TDR and TDT for differential delay lines with serpentine and flat spiral layout design. Signal waveforms and eye diagrams of four basic routing schemes are obtained by the detailed HSPICE simulation. It is found that the combination of differential signaling and flat spiral layout can exhibit the best delay line performance.
\end{abstract}

\section{Introduction}

As the cycle time of computer systems falls into the sub-nanosecond regime, the fraction of cycle time to accommodate the clock skew for synchronization of the clock signal among the logic gates has risen. While several approaches have been proposed to minimize the clock skew, delay lines are usually employed in the critical nets of a printed circuit board, e.g., serpentine or flat spiral delay lines shown in Fig.1. However, the crosstalk induced by those closely packed transmission line sections may cause a drastic deterioration in the total time delay and even result in false switching of logic gates, especially for serpentine delay line [1], [2].

With the advent of high-speed digital era, differential signaling becomes a common wiring approach because of its higher noise immunity and EMI reduction. This paper generalizes the study to differential delay lines in both serpentine and flat spiral patterns as shown in Fig.2. The TDR/TDT waveforms and TDT eye diagrams are simulated and presented to investigate the improvement in the signal integrity and noise margin.

\section{Crosstalk Effect on TDR/TDT Waveforms}

It is known that the near-end crosstalk between closely packed transmission lines in single serpentine delay line will accumulated in TDT waveform to result in laddering wave and deteriorate the time delay of signal [1]. The employment of flat spiral layout pattern has the ability to evenly spread the crosstalk noise in time and avoids the crosstalk penalty at the receiving end [2], but its far-end crosstalk will accumulate at the sending end to cause an aggravated TDR waveform. The simulated waveforms in Fig.(a) reveals the effects of crosstalk penalty, in which lossless transmission line is used in simulation.

In case of differential serpentine and flat spiral delay lines where the differential impedance is designed as $100 \Omega$, Fig.3(b) shows the simulated TDR and TDT waveforms. It is found that crosstalk induced noise is greatly reduced due to the cancellation from positive and negative voltages in differential signaling.

Quantitatively, the magnitude of near-end and far-end crosstalk can be formulated as [3]

$$
V_{\text {(near end) }}=\frac{V_{\text {(input) }}}{4}\left(\frac{L_{m}}{L_{s}}+\frac{C_{m}}{C_{s}}\right), \quad V_{\text {(far end) }}=-\frac{V_{\text {(input) }} \times T_{D}}{2 T_{r}}\left(\frac{L_{m}}{L_{s}}-\frac{C_{m}}{C_{s}}\right)
$$

For differential pairs, consider the four-conductor system (\#1, \#2, \#3, \#4) shown in Fig.2(c). Let the capacitance matrix be

$$
Q_{i}=\sum_{j=1}^{4} C_{i j} \cdot V_{j} ; i, j=1,2,3,4
$$

Consider conductors $\# 1$ and $\# 2$ are driven a differential signaling with $V_{2}=-V_{1}, Q_{2}=-Q_{1}$,

The work was supported in part by the National Science Council, Republic of China, under Grant NSC 91-2213-E-002-109. 
while conductors \#3 and \#4 are the victim lines. By a simple calculation, the self capacitance of the differential signal pair is $\left(C_{11}+C_{22}-C_{12}-C_{21}\right) / 2$ while the mutual capacitance between differential signal pair and conductor $\# 3$ is $C_{31}-C_{32}$ and conductor $\# 4$ is $C_{41}-C_{42}$. Similarly, the self inductance of the differential signal pair is $\left(L_{11}+L_{22}-L_{12}-L_{21}\right) / 2$, while the mutual inductance is $L_{31}-L_{32}$ to conductor $\# 3$ and $L_{41}-L_{42}$ to conductor \#4. Inserting the self and mutual capacitance and inductance into (1), the amount of crosstalk noise can be calculated and shown in Fig. 3(b).

Owing to the capability in crosstalk reduction by differential signaling, there is almost no difference in time delay of signal between both differential delay lines. Nevertheless, the magnitude of crosstalk noise still influences the noise margin and will be discussed in next section.

\section{Comparison of TDT Eye Diagrams}

In the simulation for eye diagrams, the pseudo random incident signal is specified with rise time 50ps, bit period 500ps (2Gbps), and voltage swing 0 IV. Figure 4 shows the simulated results for both of the differential delay lines shown in Fig.2. It is apparent that the eye of Fig.4(a) is better than that of Fig.4(b) due to the spreading effects of crosstalk noise in flat spiral delay line.

When the conductor and substrate loss is taken into consideration, Fig.5 shows the simulated eye diagram. All eye diagrams are similar to those of lossless cases. It reveals that lossy effect of transmission line is not a key factor in affecting these eye diagrams.

Figure 6 investigates the influence of the number of transmission line sections, which affects the accumulation of crosstalk, and the spacing between the sections, which affects the coupling strength. The number of sections is changed from seven to five in Fig.6(a) and the spacing from $\mathrm{s}=0.8 \mathrm{~mm}$ to $1.6 \mathrm{~mm}$ in Fig.6(b). From the figure, it can be seen that eye diagrams are better if the number of sections or the spacing between the sections is smaller.

In addition, the bit period of signal plays an important role. Figure 7 shows the simulated eye diagrams with fixed rise time but different bit period as a parameter. It can be seen that the smaller the bit period, the worse the eye diagrams.

\section{Conclusions}

In single serpentine delay line, the magnitude of the laddering wave may grow up to a significant level before the arrival of the main signal. Using single flat spiral delay line can greatly alleviate this crosstalk penalty in TDT, but with the deteriorated TDR waveform as a tradeoff. This paper extends the analysis to differential signals and finds that the crosstalk induced noise can be greatly reduced in both TDR and TDT waveforms. The combination of flat spiral routing and differential signaling can improve the signal integrity furthermore. In addition, the number of sections, the spacing between two sections, and the bit period of signal are major parameters in determining the signal integrity and should be carefully considered in the design of delay line.

\section{References}

[1] R. B. Wu and F. L. Chao, "Laddering wave in serpentine delay line," IEEE Trans. Compon. Packag., Manufact. Technol., vol. 18, pp. 644-650, Nov.1995.

[2] R. B. Wu and F. L. Chao, "Flat spiral delay line design with minimum crosstalk penalty," IEEE Trans. Compon. Packag., Manufact. Technol., vol. 19, pp.397-402, May 1996.

[3] H. Hall, W. Hall, and A. Mccall, High-Speed Digital System Design. Chap. 3, pp.48.

[4] B. J. Rubin and B. Singh, "Study of meander line delay in circuit boards," IEEE Trans. Microwave Theory Tech., vol. 48, pp.1452-1460, Sept. 2000.

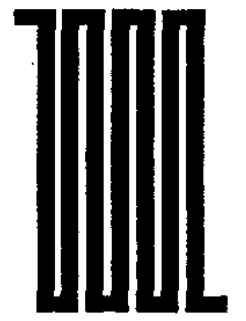

(a)

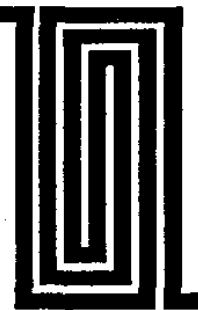

(b)

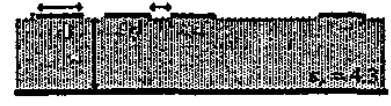

(c)

Fig.1 Single delay lines: (a) serpentine, (b) flat spiral, and (c) cross-sectional view 


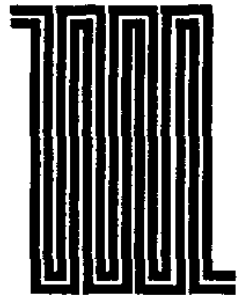

(a)

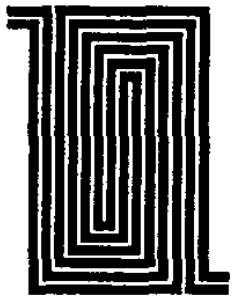

(b)

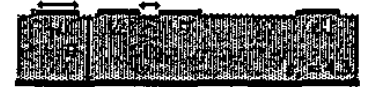

(c)

Fig.2 Differential delay lines: (a) serpentine, (b) flat spiral, and (c) cross-sectional view

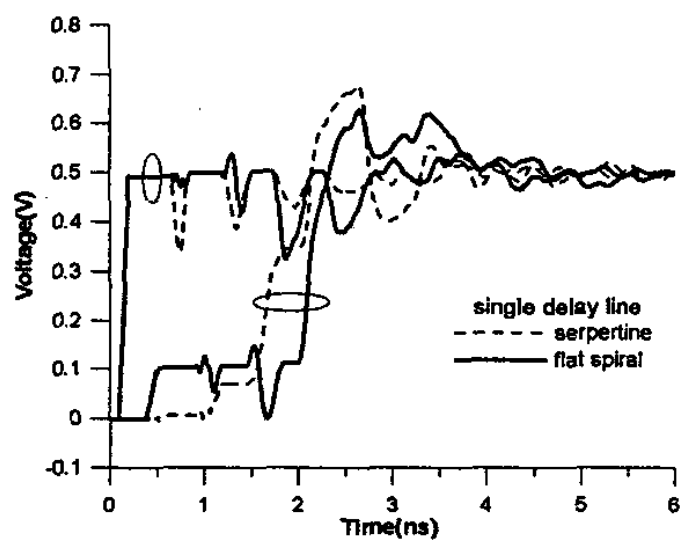

(a)

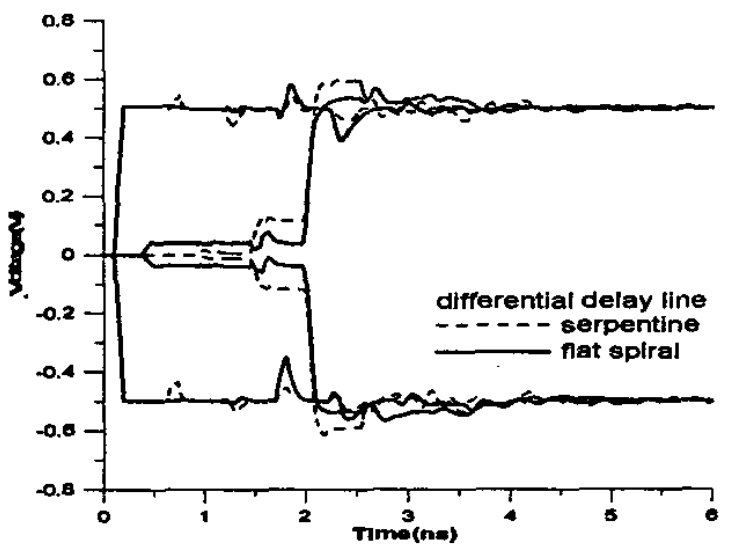

(b)

Fig.3 Simulated TDR and TDT waveforms of (a) single and (b) differential delay lines

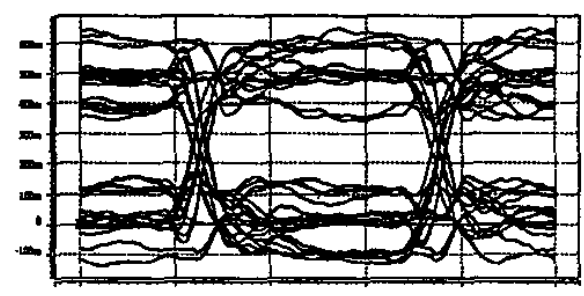

(a)

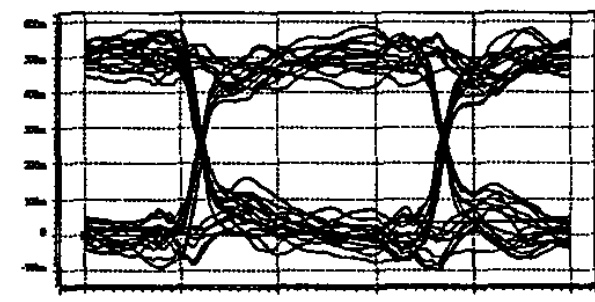

(b)

Fig.4 TDT Eye diagram of differential delay lines (lossless): (a) serpentine and (b) flat spiral

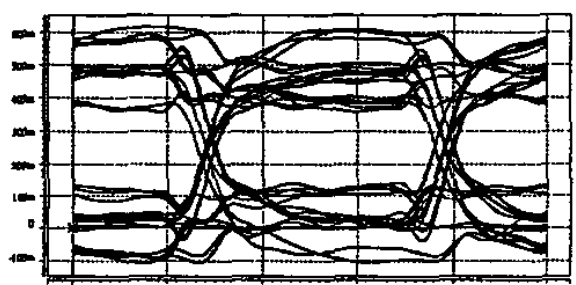

(a)

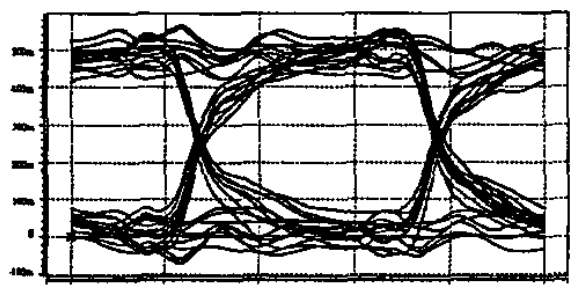

(b)

Fig.5 TDT Eye diagram of differential delay lines (lossy): (a) serpentine and (b) flat spiral 

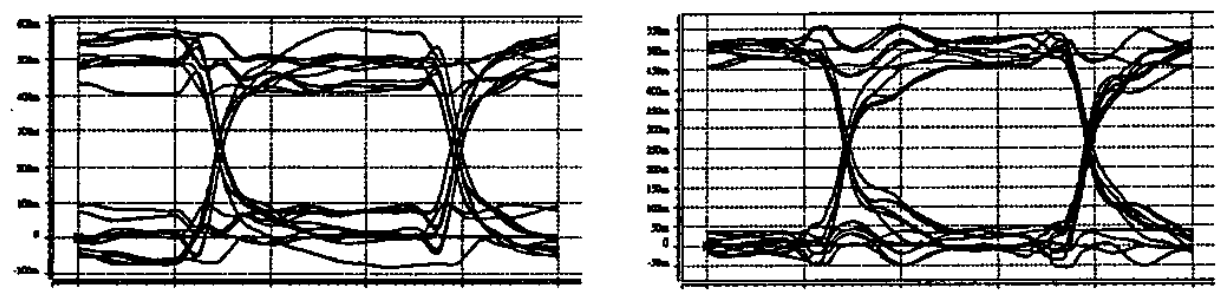

(a) five sections (left: serpentine, right: flat spiral)
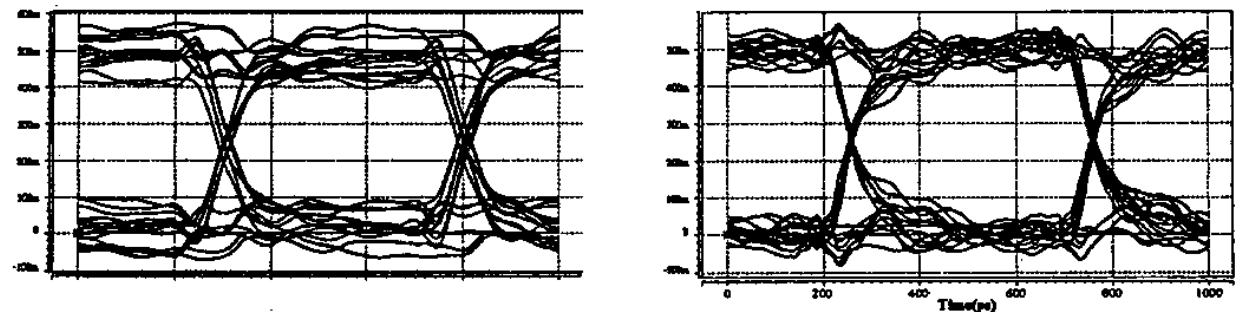

(b) $\mathrm{S}=1.6 \mathrm{~mm}$ (left: serpentine, right: flat spiral)

Fig.6 TDT Eye diagram with some structure parameters in Fig. 2 changed
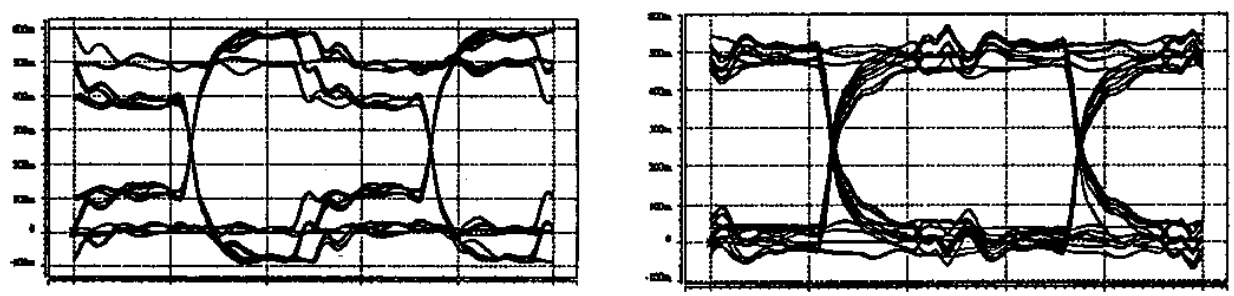

(a) bit period=1000ps (left: serpentine, right: flat spiral)
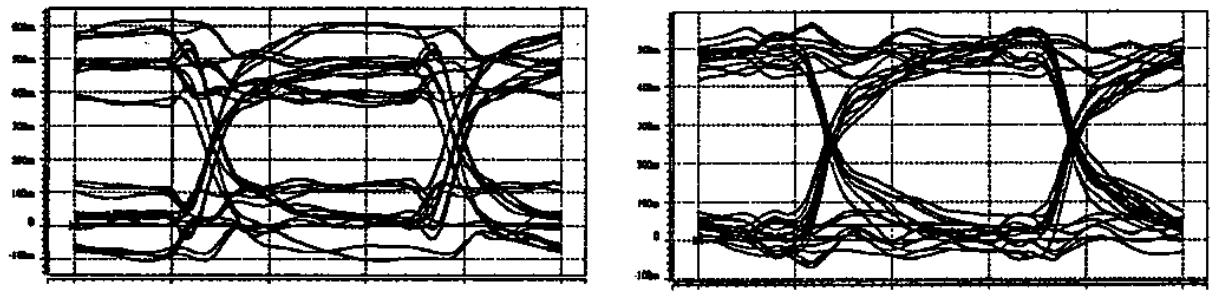

(b) bit period=500ps (left: serpentine, right: flat spiral)
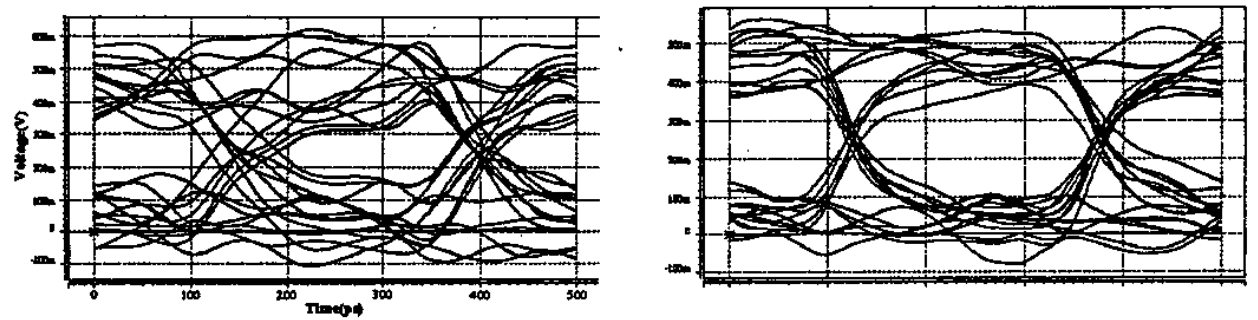

(c) bit period=250ps (left: serpentine, right: flat spiral)

Fig. 7 TDT Eye diagrams with rise time fixed at 50ps and different bit period as a parameter. 